\title{
A BOUNDARY VALUE PROBLEM OF ORTHOTROPIC ELECTRO- ELASTIC CIRCULAR CYLINDER
}

\author{
István Ecsedi \\ professor emeritus, Institute of Applied Mechanics, University of Miskolc \\ Miskolc-Egyetemváros, 3515 Miskolc, Hungary,e-mail:mechecs@uni-miskolc.hu \\ Ákos József Lengyel \\ assistant professor, Institute of Applied Mechanics, University of Miskolc \\ Miskolc-Egyetemváros, 3515 Miskolc, Hungary, e-mail: mechlen@uni-miskolc.hu \\ Attila Baksa \\ associate professor, Institute of Applied Mechanics, University of Miskolc \\ Miskolc-Egyetemváros, 3515 Miskolc, Hungary,e-mail: mechab@uni-miskolc.hu
}

\begin{abstract}
A boundary value problem of orthotropic piezoelectric solid circular cylinder which is in the state of antiplane shear deformation is studied. The whole boundary surface is loaded by an equilibrium axial traction. This paper gives an analytical solution of the considered antiplane shear deformation.
\end{abstract}

Keywords: antiplane shear deformation, piezoelectric, circular cylinder

\section{Introduction}

The antiplane shear deformation is a special case of the state of deformation of a solid body. This state is achieved when the displacements in the body are zero in the plane of interest but non-zero in the direction perpendicular to the plane. If the plane of antiplane shear deformation is the plane $O x y$ of the rectangular Cartesian coordiante system $O x y z$ and the displacement vector can be represented as $\mathbf{u}=u \mathbf{e}_{x}+v \mathbf{e}_{y}+w \mathbf{e}_{z}$. Here, $\mathbf{e}_{x}, \mathbf{e}_{y}$ and $\mathbf{e}_{z}$ are unit vectors in $x, y$ and $z$ directions, then the antiplane shear deformation is defined by the next equations $[1,2]$

$$
u=0, \quad v=0, \quad w=w(x, y) .
$$

It means that if we consider a circular cylindrical body (Fig. 1) whose generators of the circular boundary surfaces are parallel to axis $z$, all cross sections of this solid body have the same deformations according to Eq. (1). The strain field of the infinitesimal antiplane shear deformation is as follows

$$
\gamma_{x z}=\frac{\partial w}{\partial x}, \quad \gamma_{y z}=\frac{\partial w}{\partial y},
$$

where $\gamma_{x z}$ and $\gamma_{y z}$ are the shearing strains, other strains vanish. The cross section of the considered circular cylinder is a circle with radius $R$ and its surface is denoted by $A$. The boundary curve of $A$ is $\partial A$ and its unit normal vector is $\mathbf{n}=n_{x} \mathbf{e}_{x}+n_{y} \mathbf{e}_{y}$ (Fig. 2). 


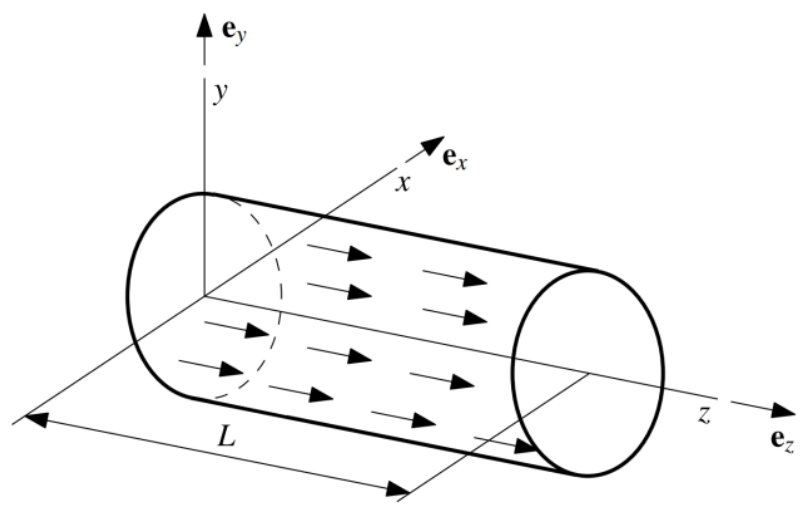

Figure 1. Piezoelectric circular cylinder.

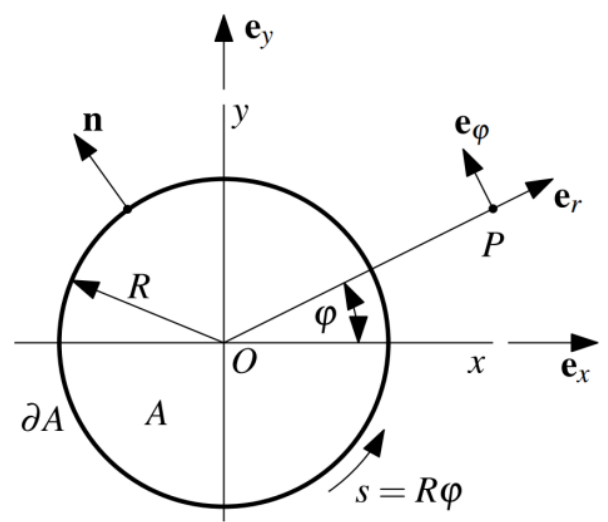

Figure 2. Cross section of the considered circular cylinder.

$$
n_{x}=\frac{x}{R}, \quad n_{y}=\frac{y}{R}, \quad x^{2}+y^{2}=R^{2} .
$$

The circular cylinder is made from orthotropic piezoelectric material [3,4]. The applied surface traction on the cylindrical boundary surface acting on axial direction is

$$
p_{z}=\frac{f}{R^{2}} x y, \quad x^{2}+y^{2}=R^{2} .
$$

The surface traction forms an equilibrium force system since we have

$$
\begin{gathered}
F_{z}=\int_{0}^{L} \int_{\partial A} p_{z} \mathrm{~d} s \mathrm{~d} z=f \frac{L}{R^{2}} \prod_{\partial A} x y \mathrm{~d} s=0, \\
M_{x}=\int_{0}^{L} \int_{\partial A} y p_{z} \mathrm{~d} s \mathrm{~d} z=f \frac{L}{R^{2}} \underset{\partial A}{\int} x y^{2} \mathrm{~d} s=0,
\end{gathered}
$$




$$
M_{y}=-\int_{0}^{L} \int_{\partial A} x p_{z} \mathrm{~d} s \mathrm{~d} z=-f \frac{L}{R^{2}} \int_{\partial A} x^{2} y \mathrm{~d} s=0 .
$$

Our aim is to determine the axial displacement $w$, the shearing stresses, the electrical potential and the electric displacement vector.

\section{Governing equations}

In the present case the next constitutive equations will be used

$$
\begin{aligned}
& \tau_{x z}=c_{55} \gamma_{x z}-e_{15} E_{x}, \\
& \tau_{y z}=c_{44} \gamma_{y z}-e_{24} E_{y}, \\
& D_{x}=e_{15} \gamma_{x z}+\kappa_{11} E_{x}, \\
& D_{y}=e_{24} \gamma_{y z}+\kappa_{22} E_{y} .
\end{aligned}
$$

In Eqs. (8-11) $E_{x}$ and $E_{y}$ are the component of electric field vector

$$
E_{x}=-\frac{\partial \phi}{\partial x}, \quad E_{y}=-\frac{\partial \phi}{\partial y}
$$

here $\phi=\phi(x, y)$ is the electric potential, furthermore $D_{x}$ and $D_{y}$ are the components of electric displacement vector, $c_{44}$ and $c_{55}$ are the elastic stiffness coefficients, $e_{15}$ and $e_{24}$ are the piezoelectric constants, $\kappa_{11}$ and $\kappa_{22}$ are the electric permittivity coefficients. In the case of antiplane shear deformation the equation of mechanical equilibrium and Gauss equation can be represented as

$$
\frac{\partial \tau_{x z}}{\partial x}+\frac{\partial \tau_{y z}}{\partial y}=0, \quad \frac{\partial D_{x}}{\partial x}+\frac{\partial D_{y}}{\partial y}=0, \quad(x, y, z) \in \partial A \times L .
$$

On the unelectroded curved boundary surface there is no free surface density, thus we have

$$
D_{x} n_{x}+D_{y} n_{y}=0, \quad(x, y, z) \in \partial A \times L .
$$

The mechanical boundary condition is as follows

$$
\tau_{x z} n_{x}+\tau_{y z} n_{y}=p_{z}, \quad(x, y, z) \in \partial A \times L,
$$

that is

$$
\tau_{x z} x+\tau_{y z} y=\frac{f}{R} x y, \quad(x, y, z) \in \partial A \times L .
$$

We are looking for the solution of boundary value problem formulated by Eqs. (8-11) and Eqs. $(13-15)$ in the next form

$$
w(x, y)=C_{w} x y, \quad \phi(x, y)=C_{\phi} x y .
$$


Ecsedi, I., Lengyel, A. J., Baksa, A. A boundary value problem of orthotropic electroelastic circular cylinder

From Eqs. (8-11) and Eq. (17) it follows that

$$
\begin{aligned}
& \tau_{x z}=\left(c_{55} C_{w}+e_{15} C_{\phi}\right) y, \\
& \tau_{y z}=\left(c_{44} C_{w}+e_{24} C_{\phi}\right) x, \\
& D_{x}=\left(e_{15} C_{w}-\kappa_{11} C_{\phi}\right) y, \\
& D_{y}=\left(e_{24} C_{w}-\kappa_{22} C_{\phi}\right) x .
\end{aligned}
$$

It is evident with arbitrary constants $C_{w}$ and $C_{\phi}$ that the equation of mechanical equilibrium and Gauss equation are satisfied. From the stress boundary conditions and electric boundary conditions we get a system of linear equations for $C_{w}$ and $C_{\phi}$

$$
\begin{aligned}
& \left(c_{55}+c_{44}\right) C_{w}+\left(e_{15}+e_{24}\right) C_{\phi}=\frac{f}{R}, \\
& \left(e_{15}+e_{24}\right) C_{w}-\left(\kappa_{11}+\kappa_{22}\right) C_{\phi}=0 .
\end{aligned}
$$

The solution of the system of linear equations (22-23) is as follows

$$
\begin{aligned}
& C_{w}=\frac{f}{R} \frac{\kappa_{11}+\kappa_{22}}{\left(c_{55}+c_{44}\right)\left(\kappa_{11}+\kappa_{22}\right)+\left(e_{15}+e_{24}\right)^{2}}, \\
& C_{\phi}=\frac{f}{R} \frac{e_{15}+e_{24}}{\left(c_{55}+c_{44}\right)\left(\kappa_{11}+\kappa_{22}\right)+\left(e_{15}+e_{24}\right)^{2}} .
\end{aligned}
$$

Substitution the expressions of $C_{w}$ and $C_{\phi}$ into Eqs. (17-21) leads to the explicit solution formula of the considered boundary value problem.

The cross-sectional shear forces $V_{x}, V_{y}$ and cross-sectonal torque $T$ can be computed as

$$
\begin{gathered}
V_{x}=\int_{A} \tau_{x z} \mathrm{~d} A=\left(c_{55} C_{w}+e_{15} C_{\phi}\right) \int_{A} y \mathrm{~d} A=0, \\
V_{y}=\int_{A} \tau_{y z} \mathrm{~d} A=\left(c_{44} C_{w}+e_{24} C_{\phi}\right) \int_{A} \mathrm{x} \mathrm{d} A=0, \\
T=\int_{A}\left(x \tau_{y z}-y \tau_{x z}\right) \mathrm{d} A=\left[\left(c_{44}-c_{55}\right) C_{w}+\left(e_{24}-e_{15}\right) C_{\phi}\right] \frac{R^{4} \pi}{4}= \\
=\frac{f}{4} R^{3} \pi \frac{\left(c_{44}-c_{55}\right)\left(\kappa_{11}+\kappa_{22}\right)+e_{24}^{2}-e_{15}^{2}}{\left(c_{44}+c_{55}\right)\left(\kappa_{11}+\kappa_{22}\right)+\left(e_{15}+e_{24}\right)^{2}} .
\end{gathered}
$$




\section{Example for the application of Betti theorem}

The solution of Saint-Venant torsion for solid circular cross section made of orthotropic electroelastic material is as follows [5]

$$
u=-\vartheta y z, \quad v=\vartheta x z, \quad w=\vartheta \omega(x, y), \quad V=\vartheta U(x, y),
$$

where $\vartheta$ is the rate of twist and $\omega=\omega(x, y)$ is the torsion function, $U=U(x, y)$ is the electric potential function for unit value of $\vartheta$. We have [5]

$$
\omega(x, y)=C_{\omega} x y, \quad U(x, y)=C_{U} x y .
$$

Here

$$
\begin{aligned}
& C_{\omega}=\frac{\left(c_{55}-c_{44}\right)\left(\kappa_{11}+\kappa_{22}\right)+e_{15}^{2}-e_{24}^{2}}{\left(c_{55}+c_{44}\right)\left(\kappa_{11}+\kappa_{22}\right)+\left(e_{15}+e_{24}\right)^{2}}, \\
& C_{U}=\frac{2\left(c_{55} e_{24}-c_{44} e_{15}\right)}{\left(c_{55}+c_{44}\right)\left(\kappa_{11}+\kappa_{22}\right)+\left(e_{15}+e_{24}\right)^{2}} .
\end{aligned}
$$

In the case of Saint-Venant torsion the curved boundary surface of the circular cylinder is stress and charge free. According to the reciprocity relation of the linear piezoelectric bodies we have in our case

$$
W_{12}=\vartheta L \int_{\partial A} p_{z} \omega \mathrm{d} s+\vartheta L \int_{A}\left(-y \tau_{x z}+x \tau_{y z}\right) \mathrm{d} A .
$$

In Eq. (33) $p_{z}$ is given by Eq. (4) and $\tau_{x z}, \tau_{y z}$ are given by Eqs. (18) and (19). The surface tractions of Saint-Venant torsion on the end cross section are in plane load which does not give any work on the axial displacement field of antiplane shear deformation. Since in the cases of Saint-Venant torsion and condisered antiplane shear deformation there are no charge free surface and charge density free per unit volume and we have $D_{z} \equiv 0$ in the whole body in our cases of antiplane deformation for Saint-Venant torsion the electric potential does not appear in Betti's theorem and we have

$$
W_{21}=0 \text {. }
$$

A detailed computation which is based on Eqs. $(5,19,20,21,25$ and 26) gives the next results

$$
\begin{gathered}
\int_{\partial A} p_{z} \omega \mathrm{d} s=-\frac{f R^{3}}{4} \frac{\left(c_{44}-c_{55}\right)\left(\kappa_{11}+\kappa_{22}\right)+e_{24}^{2}-e_{15}^{2}}{\left(c_{44}+c_{55}\right)\left(\kappa_{11}+\kappa_{22}\right)+\left(e_{15}+e_{24}\right)^{2}} \pi, \\
\int_{A}\left(x \tau_{y z}-y \tau_{x z}\right) \mathrm{dA}=\frac{f R^{3}}{4} \frac{\left(c_{44}-c_{55}\right)\left(\kappa_{11}+\kappa_{22}\right)+e_{24}^{2}-e_{15}^{2}}{\left(c_{44}+c_{55}\right)\left(\kappa_{11}+\kappa_{22}\right)+\left(e_{15}+e_{24}\right)^{2}} \pi .
\end{gathered}
$$

From Eqs. (35) and (36) it follows that according to Betti's theorem we have in the present case

$$
W_{12}=W_{21}=0 \text {. }
$$




\section{Numerical example}

In the numerical example the next data are used: $c_{44}=6.27 \times 10^{10} \mathrm{~Pa}, c_{55}=5.13 \times 10^{10} \mathrm{~Pa}$, $e_{15}=17 \mathrm{C} / \mathrm{m}^{2}, \quad e_{24}=9 \mathrm{C} / \mathrm{m}^{2}, \quad \kappa_{11}=2.0797 \times 10^{-9} \mathrm{C} / \mathrm{Vm}, \quad \kappa_{22}=2.186 \times 10^{-9} \mathrm{C} / \mathrm{Vm}$, $f=20 \mathrm{MPa}, R=0.05 \mathrm{~m}$.

Fig. 3 shows the contour plot of $w=w(x, y)$ and the three-dimensional plot of axial displacements as given in Fig. 4. Fig. 5 and Fig. 6 illustrate the contour plot and three-dimensional plot of electric potential function $\phi=\phi(x, y)$. The three-dimensional plot of the function

$$
\tau(x, y)=\tau_{r z}(x, y)-p_{z}(x, y)
$$

is shown in Fig. 7. This figure also illustrates that on the cylindrical boundary surface segment $\tau(x, y)=0$ according to the stress boundary condition (15).

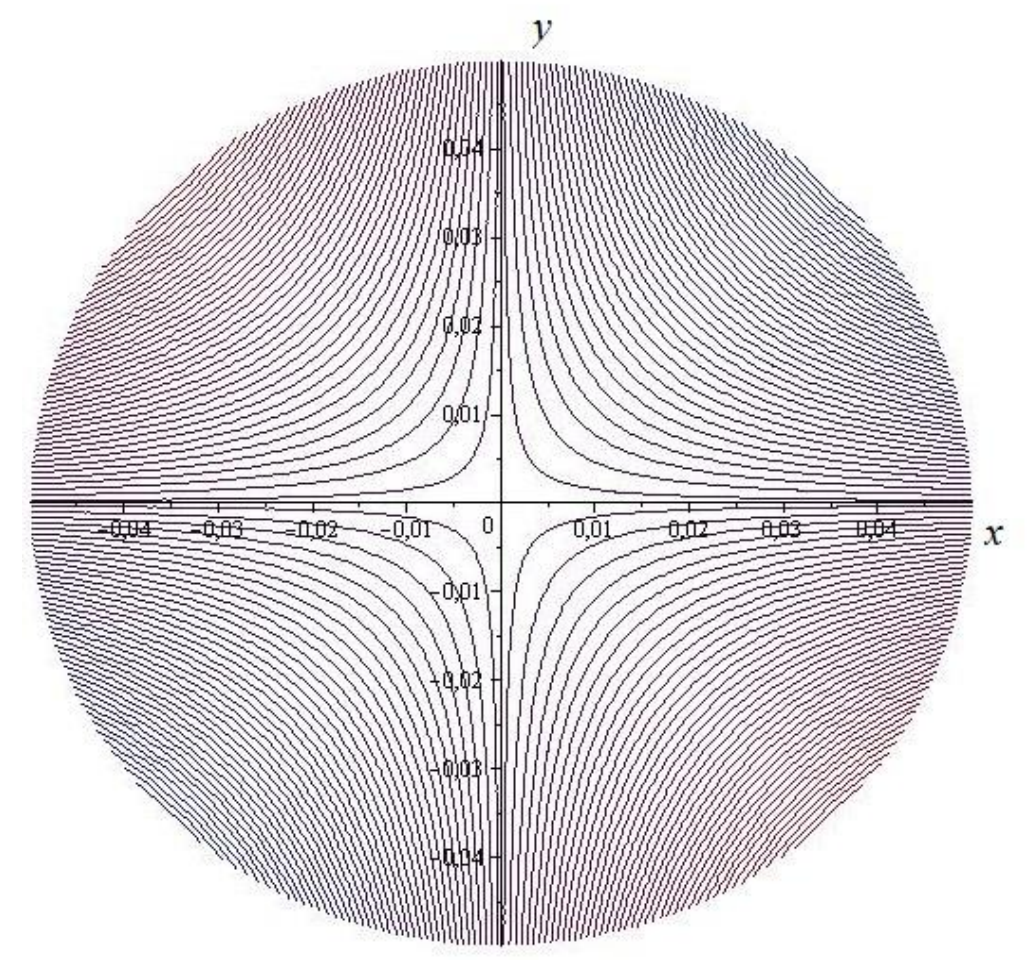

Figure 3. Contour plot of $w=w(x, y)$. 


\section{Ecsedi, I., Lengyel, A. J., Baksa, A. A boundary value problem of orthotropic electroelastic circular cylinder}

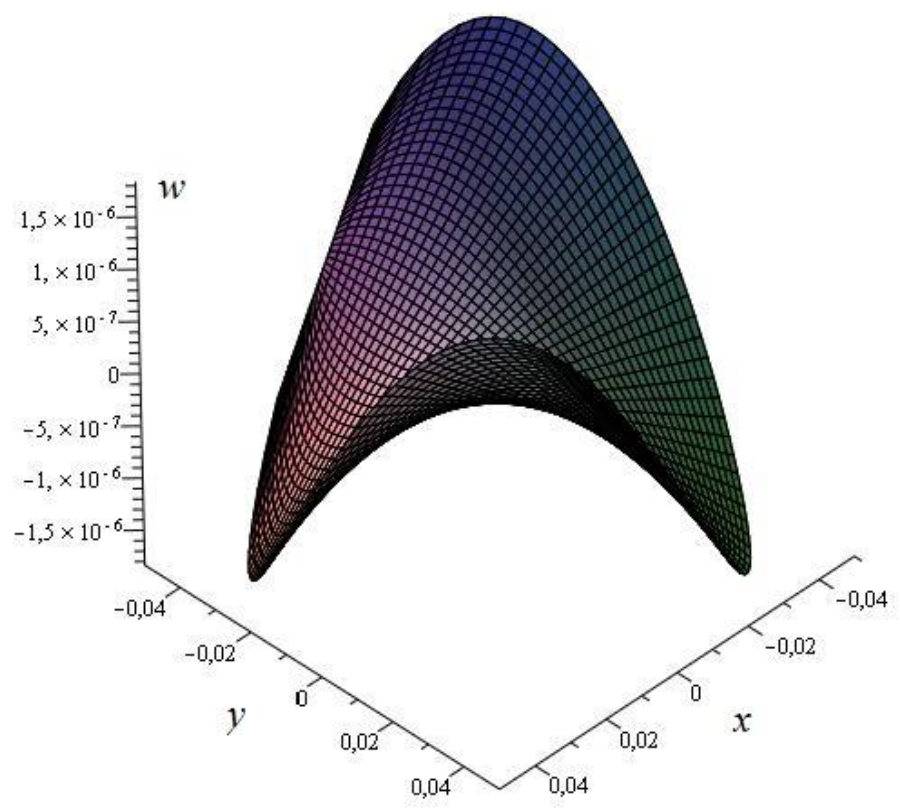

Figure 4. Three-dimensional plot of $w=w(x, y)$.

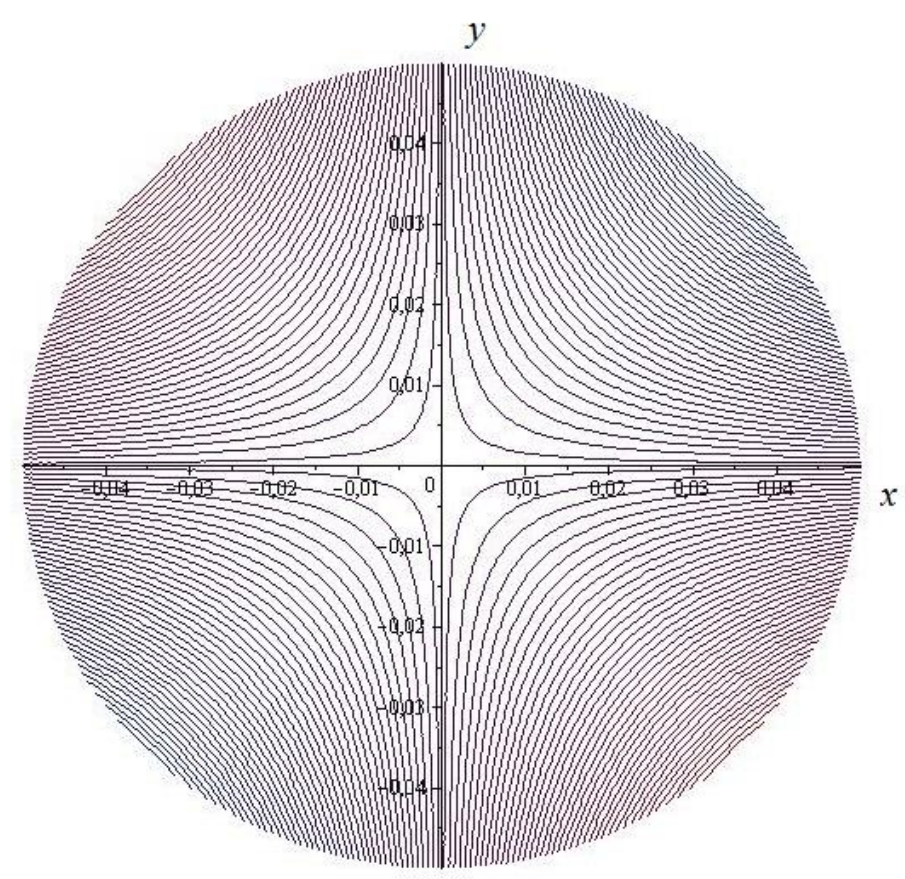

Figure 5. Contour plot of $\phi=\phi(x, y)$. 


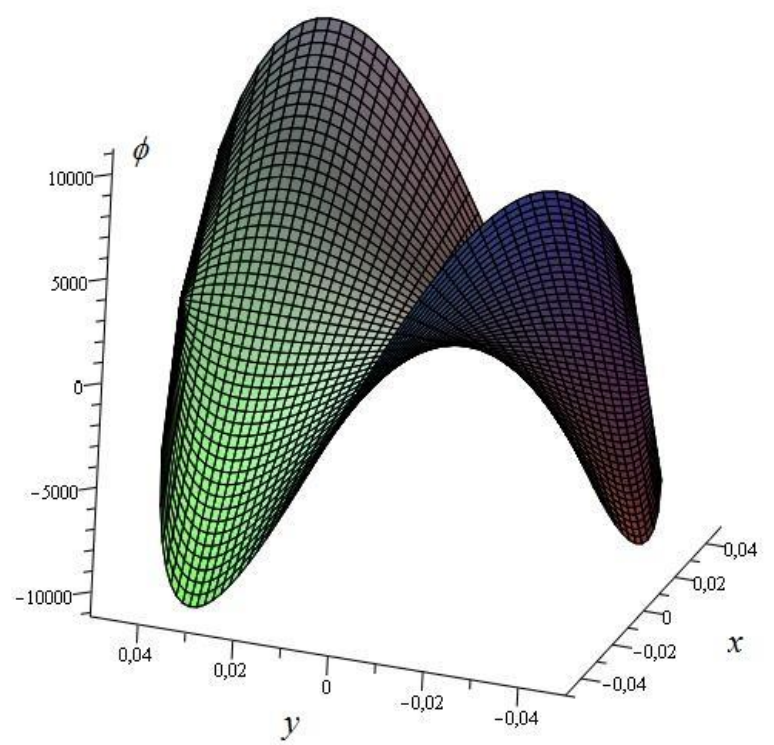

Figure 6. Three-dimensional plot of $\phi=\phi(x, y)$.

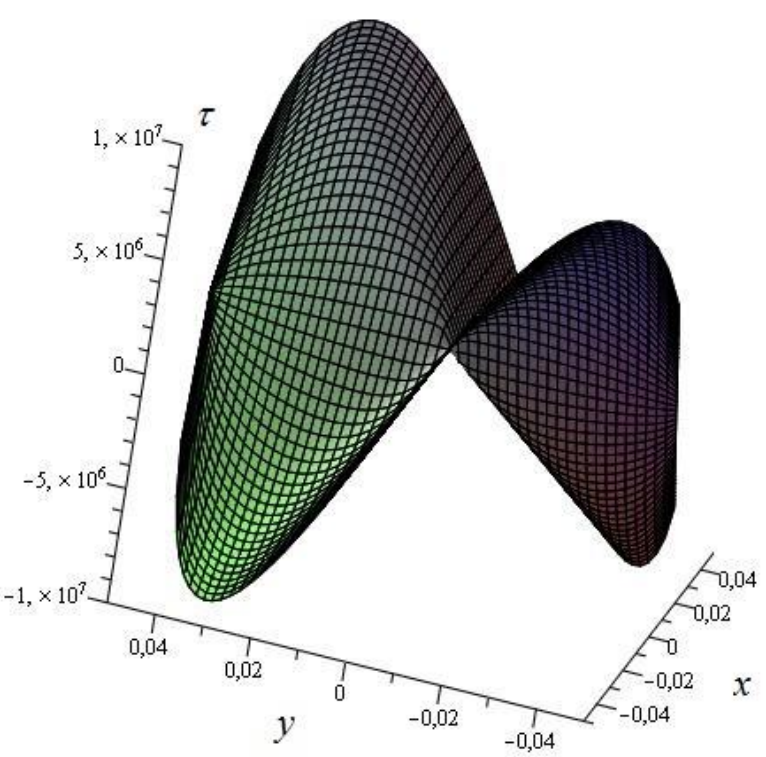

Figure 7. Three-dimensional plot of $\tau_{r z}(x, y)-p_{z}(x, y)$. 
Ecsedi, I., Lengyel, A. J., Baksa, A. A boundary value problem of orthotropic electroelastic circular cylinder

\section{Acknowledgements}

This research was supported by the National Research, Development and Innovation Office - NKFIH, K115701.

This research was also carried out as part of the EFOP-3.6.1-16-2016-00011 Younger and Renewing University - Innovative Knowledge City - institutional development of the University of Miskolc aiming at intelligent specialisation" project implemented in the framework of the Szechenyi 2020 program. The realization of this project is supported by the European Union, co-financed by the European Social Fund.

\section{References}

[1] Ting, T. C. T. (1996). Anisotropic Elasticity. Theory and Applications. Oxford University Press, Oxford. https://doi.org/10.1093/oso/9780195074475.001.0001

[2] Barber, J. R. (2010). Elasticity. Springer-Verlag, Berlin. https://doi.org/10.1007/978-90-481-3809-8

[3] Yang, J. (2005). An introduction to the theory of piezoelectricity. Springer-Verlag, New York.

[4] Yang, J. (2006). Mechanics of piezoelectric structures. World Scientific, New York. https://doi.org/10.1142/6057

[5] Ecsedi, I., Baksa, A. (2012). A variational formulation for the torsional problem of piezoelastic beams. Applied Mathematical Modelling, 36(4), 1668-1677.

https://doi.org/10.1016/j.apm.2011.09.021 